\title{
DECISIONS
}

\section{A young woman with acute one-sided facial paralysis}

\author{
Ildikó Gagyor MD, Vishnu Madhok MBChB, Frank Sullivan PhD
}

A 24-year-old woman presents in the emergency department with weakness on the left side of her face that started two hours previously. She reports a sudden onset of an uncomfortable sensation in the affected region with difficulty closing her left eye, a crooked smile and increased sensitivity to sound. The examination shows unilateral peripheral facial paralysis with features of a lower motor neuron lesion.

\section{What diagnosis should be considered?}

The primary diagnosis to consider is Bell palsy, an idiopathic paresis of the muscles supplied by the facial nerve (seventh cranial nerve). The main differential diagnosis for this patient includes Lyme disease and herpes zoster oticus (Ramsay Hunt syndrome), or some rarer viral cause such as herpes simplex, mumps or cytomegalovirus. Much more rare conditions include other infectious diseases (e.g., meningitis, otitis media and HIV infection), autoimmune diseases (e.g., sarcoidosis, Guillain-Barré syndrome and Melkersson-Rosenthal syndrome) and tumours of the parotid gland or facial nerve. These conditions often have associated symptoms and signs that help distinguish them from Bell palsy, such as fever, malaise, rash, arthralgia and pain. ${ }^{1,2}$

\section{What is required on physical examination?}

The diagnosis of Bell palsy is made clinically. ${ }^{3}$ Patients present with acute-onset unilateral facial weakness and are unable to completely close the eye on the affected side. The main diagnostic challenge is to be certain the cause is not in the central nervous system. A central lesion would result in a pattern of weakness suggesting an upper motor neuron lesion, with sparing of the forehead owing to bilateral innervation of these muscles. The severity of the lesion can be assessed using several methods, including the House-Brackmann scale (grade $\mathrm{I}=$ normal facial function, grade VI $=$ total paralysis). ${ }^{4}$ The auditory canal should also be investigated for vesicles to exclude herpes zoster.

\section{What tests should be performed?}

Blood tests are usually not indicated. To rule out Lyme disease in endemic areas, a history of tick exposure, erythema migrans or arthralgia should be explored first; if necessary, a blood sample should be sent for serologic testing before starting treatment. If an infection is suspected, measurement of inflammatory markers (complete blood count, erythrocyte sedimentation rate and C-reactive protein) should be ordered. ${ }^{2}$ Radiologic imaging is not needed to establish the diagnosis unless there are worrying features suggesting a tumour, such as swelling of the parotid gland or signs of raised intracranial pressure.

\section{What treatments should be started?}

Corticosteroids are the first line of treatment in the acute phase (within $72 \mathrm{~h}$ after symptom onset) because they have shown a significant benefit for patients with Bell palsy compared with placebo, on the basis of evidence from two large randomized controlled trials (RCTs) and a subsequent systematic review..$^{5-7}$ In one trial, patients given $25 \mathrm{mg}$ of prednisolone twice daily for up to 10 days had lower rates of incomplete recovery than those who did not receive prednisolone (5.6\% v. 8.4\%). ${ }^{5}$ The number needed to treat with corticosteroids to achieve one additional complete recovery, compared with no treatment, was six after three months and eight after nine months. ${ }^{5}$ Combination treatment with a corticosteroid and an antiviral agent (acyclovir, famciclovir or valacyclovir) may be promising, as shown in a systematic review and meta-analysis of $11 \mathrm{RCTs},{ }^{8}$ but this treatment approach requires further study. Antiviral treatment alone is not recommended, because it has not shown a positive effect on recovery. ${ }^{8}$

Eye protection should be provided for patients who cannot close their eye completely, as well as lubricating drops and ointments to protect against corneal ulcers. ${ }^{5,6}$

A decision-making algorithm from the guideline on Bell palsy from the Canadian Society of Otolaryngology - Head and Neck Surgery and
Competing interests: None declared.

This article has been peer reviewed.

The clinical scenario is fictional.

Correspondence to: Ildikó Gagyor,igagyor@gwdg.de

CMAJ 2016. DOI:10.1503 /cmaj.160108 
Canadian Neurological Sciences Federation ${ }^{9}$ is available in Appendix 1 (www.cmaj.ca/lookup/ suppl/doi:10.1503/cmaj.160108/-/DC1).

\section{What should the patient be told about the prognosis?}

The prognosis for Bell palsy, even if left untreated, is good for most patients. However, about $30 \%$ of those who are untreated will have a poor recovery, with continuing facial disfigurement, psychological difficulties and facial pain. ${ }^{10}$ As described earlier, treatment with corticosteroids greatly increases the rate of complete recovery by $31 \%$ and reduces the incidence of sequelae such as motor synkinesis and crocodile tears by $44 \%{ }^{8}$

\section{Should the patient be referred?}

Patients with Bell palsy can be managed confidently in family practice or as outpatients. If there are features (e.g., diplopia, bilateral palsy, weakness of the contralateral side, signs of infection) suggestive of another cause, poor recovery or progressive weakness, a referral to a specialist for imaging and to rule out other causes of facial weakness will be required. ${ }^{9}$

\section{Case revisited}

The patient was found to have Bell palsy after other reasons for facial paresis were excluded, and she received treatment within 72 hours after symptom onset. Treatment consisted of $60 \mathrm{mg}$ prednisolone daily for 10 days. Facial weakness was assessed as grade IV at onset, involving incomplete closure of the eye lid. Protective eye drops and ointments were prescribed. A complete recovery was achieved four weeks after presentation. There were no sequelae.

\section{References}

1. Gilden DH. Bell's palsy. N Engl J Med 2004;351:1323-31

2. Patel DK, Levin KH. Bell palsy: Clinical examination and management. Cleve Clin J Med 2015;82:419-26.
3. Bharathi R, Sullivan F, Aliarzadeh B, et al. Validation of identification of Bell's palsy cases in Canadian primary care EMR data - a pilot study. Ann Otolaryngol Rhinol 2016;3:1082.

4. Fattah AY, Gurusinghe AD, Gavilan J, et al. Facial nerve grading instruments: systematic review of the literature and suggestion for uniformity. Plast Reconstr Surg 2015;135:569-79.

5. Sullivan FM, Swan IR, Donnan PT, et al. Early treatment with prednisolone or acyclovir in Bell's palsy. N Engl J Med 2007;357: 1598-607.

6. Engström M, Berg T, Stjernquist-Desatnik A, et al. Prednisolone and valaciclovir in Bell's palsy: a randomised, double-blind, placebocontrolled, multicentre trial. Lancet Neurol 2008;7:993-1000.

7. Madhok VB, Gagyor I, Daly F, et al. Corticosteroids for Bell's palsy (idiopathic facial paralysis). Cochrane Database Syst Rev 2016;(7):CD001942

8. Gagyor I, Madhok VB, Daly F, et al. Antiviral treatment for Bell's palsy (idiopathic facial paralysis). Cochrane Database Syst Rev 2015;(11):CD001869.

9. de Almeida JR, Guyatt GH, Sud S, et al. Management of Bell palsy: clinical practice guideline. CMAJ 2014;186:917-22.

10. Peitersen E. Bell's palsy: the spontaneous course of 2,500 peripheral facial nerve palsies of different etiologies. Acta Otolaryngol Suppl 2002;(549):4-30.

Affiliations: Department of General Practice (Gagyor), University Medical Center Göttingen, Göttingen, Germany; Park House Surgery (Madhok), Bagshot, UK; Family Medicine Teaching Unit, North York General Hospital (Sullivan), Toronto, Ont.; Department of Family and Community Medicine and Dalla Lana School of Public Health (Sullivan), University of Toronto, Toronto, Ont.; Institute for Clinical Evaluative Sciences (Sullivan), Toronto, Ont.

Contributors: All of the authors contributed substantially to the conception and design of the manuscript, drafted and revised it critically for important intellectual content, approved the final version to be published and agreed to act as guarantors of the work.

Decisions is a series that focuses on practical evidence-based approaches to common presentations in primary care. The articles address key decisions that a clinician may encounter during initial assessment. The information presented can usually be covered in a typical primary care appointment. Articles should be no longer than 650 words, may include one box, figure or table and should begin with a very brief description (75 words or less) of the clinical situation. The decisions addressed should be presented in the form of questions. A box providing helpful resources for the patient or physician is encouraged. 